\title{
ORCHESTRATED RESTRUCTURING EVENTS DURING SECRETORY GRANULE MATURATION MEDIATE INTRAGRANULAR CARGO SEGREGATION
}

\section{Zulfeqhar A. Syed ${ }^{1}$, Liping Zhang ${ }^{1}$, Duy T. Tran ${ }^{2}$, Christopher K. E. Bleck ${ }^{3}$ and Kelly G. Ten Hagen $^{1 *}$}

${ }^{1}$ Developmental Glycobiology Section, NIDCR, National Institutes of Health, 30 Convent Drive, Bethesda, Maryland 20892-4370; ${ }^{2}$ NIDCR Imaging Core, NIDCR, National Institutes of Health, Bethesda, Maryland 20892-4370; ${ }^{3}$ Electron Microscopy Core Facility, National Heart, Lung, and Blood Institute, 10 Center Drive, Bethesda, MD 20892

*To whom correspondence should be addressed: Kelly G. Ten Hagen, Ph.D., Building 30, Room 407, 30 Convent Drive, MSC 4370, Bethesda, MD 20892-4370. Tel: 301-451-6318; Fax: 301-402-0897; Email: Kelly.Tenhagen@nih.gov

Running title: Intragranular cargo segregation during secretory granule maturation

Key words: mucin/secretion/secretory granules/O-glycosylation/Drosophila/salivary gland/ER/Golgi/calcium 


\begin{abstract}
Regulated secretion is an essential process where proteins are packaged into membranous secretory vesicles. However, the details of cargo packaging and secretory granule maturation are largely unknown. Here, we demonstrate that multiple distinct proteins undergo orchestrated intragranular restructuring during secretory granule maturation in vivo, to allow spatial segregation of distinct components within the same granule. Furthermore, through a combination of genetics and multimodality imaging, we demonstrate the molecular identity of each distinct intragranular structure. We further identify genes that are essential for the temporally-ordered restructuring events, including those controlling $\mathrm{pH}$ (vha16.1), $\mathrm{Cl}^{-}$ions (Clic and $\mathrm{ClC}-\mathrm{c}$ ) and $\mathrm{Ca}^{2+}$ ions (fwe). Finally, we show that altered cargo glycosylation influences dimensions of these structures, thereby affecting secretory granule morphology. This study elucidates key steps and factors involved in intragranular, rather than intergranular, segregation of cargo through regulated restructuring events during secretory granule maturation. Understanding how multiple distinct proteins are efficiently packaged into and secreted from the same secretory granule may provide insight into diseases resulting from defects in secretion.
\end{abstract}




\section{INTRODUCTION}

Regulated secretion is an essential process where proteins are packaged into membranous secretory granules that then await a signal to deliver their contents to the extracellular space. While diverse cells and tissues undergo regulated secretion, the details of how specific proteins are transported through the secretory apparatus, appropriately modified and then subsequently targeted to and packaged within secretory granules remain largely unknown. Two basic models have been proposed where cargo is sorted into secretory granules either by "sorting for entry" or by "selective retention" ${ }^{1}$, along with other models of unconventional protein secretion ${ }^{2}$. In the bag cells of Aplysia californica, pro-egg laying hormone undergoes endoproteolytic cleavage in the Golgi complex to yield two peptides which are then sorted into two distinct vesicles and transported to different sites in the cell ${ }^{3,4,5}$. Likewise, stratified squamous epithelia produce lamellar granules (LG) that contain distinct components that are independently trafficked to the cell surface ${ }^{6}$. In contrast, in neuronal large dense-core vesicles (LDCVs), multiple components are heterogeneously distributed in a secretory granule and co-transported $7,8,9,10,11,12,13$. When many proteins do reside within the same secretory granule, questions arise regarding how these diverse proteins are organized and segregated, so that they may be appropriately secreted upon fusion of the granule with the plasma membrane of the cell. The issues of protein transport, compaction, segregation and secretion are of particular importance for large extracellular matrix proteins, many of which undergo regulated hydration and expansion as they are secreted from specialized cells ${ }^{14,15,16,17,18,19}$.

Mucins are a family of large, highly O-glycosylated apical extracellular matrix proteins that line and protect epithelia surfaces ${ }^{20,21}$. Within the mammalian digestive tract, the predominant intestinal mucin MUC2, is thought to undergo a regulated unfolding process during secretion that results in the formation of a hydrated layer that expands to line and protect epithelial cells ${ }^{15,21,22}$. In vitro studies using N-terminal portions of MUC2 suggest that $\mathrm{pH}$-dependent interactions regulate the formation of supramolecular MUC2 structures ${ }^{16,23,24}$. Based on these in vitro studies, one model suggests that $\mathrm{C}$-terminal disulfide bonding along with $\mathrm{Ca}^{2+}$-dependent charge shielding may result in the compaction of MUC2 complexes into stack rings within secretory granules ${ }^{16}$, while an alternative model suggests that MUC2 complexes exist as linear polymers ${ }^{24}$. Similar in vitro folding studies with truncated portions of the pulmonary mucin, MUC5B, have demonstrated calcium and $\mathrm{pH}$ dependent $\mathrm{N}$ - and $\mathrm{C}$-terminal interactions that are proposed to result in the formation of compacted polymers arranged around a central node ${ }^{25,26,27}$. However, whether fulllength, native MUC2 or MUC5B form such structures within secretory granules in vivo and how this compaction process is orchestrated remain unknown. Also, whether multiple large proteins can be efficiently packaged and appropriately separated within the same secretory granule remains unknown. 
Drosophila salivary glands (SGs) are the major secretory organ in the fly and synthesize multiple highly O-glycosylated mucins that are packaged and secreted in response to developmentally-regulated hormone pulses $28,29,30,31,32$. The size of the glands and their secretory structures, in addition to an array of the fluorescently-labeled proteins, have allowed visualization of early stages of secretory granule formation through final secretion of protein products ${ }^{33,34,35}$. By following a fluorescently-labeled mucin (Sgs3-GFP; ${ }^{33}$ ) in the SGs, it has been shown that secretory granules biosynthesis is dependent on the conserved ER/Golgi transmembrane protein Tango1, which forms functional contact/docking sites between the ER and Golgi, through which cargo proteins transit ${ }^{36,37}$. Once in the Golgi apparatus, cargo proteins are modified/glycosylated and then packaged into small secretory granules that bud from the trans-Golgi network (TGN) in a clathrin and AP-1 dependent fashion ${ }^{36,38}$. These small immature secretory granules then undergo homotypic fusion ${ }^{36,38,39,40}$ and maturation steps that are dependent on endosomally-derived proteins ${ }^{41,42}$ to form large granules that are between 3-8 $\mu \mathrm{M}$ in diameter ${ }^{38,39,40}$. These large secretory granules remain in the cytoplasm awaiting a second large hormone pulse, at which time the granules fuse with the plasma membrane and secrete their contents into the lumen of the SGs in an actomyosin-dependent process ${ }^{34,35}$. However, it is not known whether the multiple distinct mucins expressed by the SGs are targeted to unique secretory granules or packaged within the same granule. Likewise, the details of cargo compaction/segregation and the factors that control it during the secretory granule enlargement/maturation phase remain unknown.

Given our current gaps in understanding how large extracellular proteins are sorted, packaged and folded within secretory granules to ensure proper secretion in vivo, we used the Drosophila SG system to address these questions. Here through genetics and multimodality imaging, we find that each secretory granule contains multiple distinct mucins that undergo organized folding/restructuring events to generate distinct structures that become spatially-segregated within mature secretory granules. Moreover, we define the endogenous mucins that comprise each distinct structure and further identify the temporally-regulated genes controlling $\mathrm{pH}$ and ion concentrations that are essential for the restructuring events occurring during the secretory granule maturation process. Our data indicate that individual mucins adopt unique structures in vivo through a regulated secretory granule maturation process controlled by calcium ions, chloride ions, $\mathrm{pH}$ and glycosylation. Furthermore, we show that intragranular restructuring and spatial segregation of each mucin, rather than intergranular separation of distinct mucins, is the predominant mechanism for cargo packaging and secretion within this organ. 


\section{RESULTS}

\section{Multiple distinct structures exist in individual secretory granules}

Using fly lines that express a fluorescently-labeled mucin (Sgs3-GFP) ${ }^{33}$, we examined SGs at various stages of development based on the presence of Sgs3-GFP containing secretory granules (Fig. 1a-d). As described previously ${ }^{33,38}$, early $3^{\text {rd }}$ instar SGs that have not yet begun to synthesize secretory cargo have no GFP present (Stage 0) (Fig. 1a). As development proceeds and the first hormone pulse occurs, SGs begin to synthesize Sgs3-GFP cargo in a spatially-regulated fashion, beginning in the distal most cells (Stage 1; Fig. 1b) and then proceeding proximally. As shown previously, small immature secretory granules then undergo homotypic fusion to form large, mature granules that are present throughout all secretory cells of the SG (Stage 2) (Fig. 1c). Finally, in response to a second hormone pulse, secretory granules fuse with the apical plasma membrane to secrete their cargo into the lumen of the SGs (Stage 3, Fig. 1d). We used SGs expressing Sgs3-GFP (Stage 2, Fig.1c,e) to further investigate the details of secretory granule biogenesis and maturation via transmission electron microscopy (TEM) (Fig. 1f). Interestingly, high magnification micrographs of individual mature granules (between 3-8 $\mu \mathrm{M}$ in diameter) reveal distinct intragranular structures (Fig. 1f, g-i). For example, the majority of the granule is occupied by bundles of electron-dense filaments that are evenly spaced apart by $\sim 28 \mathrm{~nm}$ (Fig. 1f, g and j). Additionally, bundles of electron-dense dots that have the same spacing seen for the filaments are also present (Fig. 1f, 1h and j), suggesting that they may represent cross sections of the filament bundles. The other distinct structures observed are electron-lucent ovals/discs that are approximately $78 \mathrm{~nm}$ in diameter (Fig. 1f, i and j) and most often seen in close proximity to an electron-dense matrix (Fig. 1i; white arrowhead ). A closer view of several of high magnification TEM micrographs of the discs shows the presence of an electron-dense node in the center (black arrowhead in Fig. 1i). Taken together, these results demonstrate the presence of at least 3 distinct structures within mature secretory granules: filament bundles, electron-dense matrix and electron-lucent discs. It has been suggested that aldehyde based fixation methods could induce structural artifacts in secretory granule morphology under EM ${ }^{43}$, However, comparison of aldehyde and cryofixed (HPF) secretory granules demonstrates that the structures seen are independent of fixation procedures (Sup.Fig. 1a and b).

To better understand the spatial distribution of the electron-lucent discs (hereafter referred to as "discs") and the electron-dense matrix, we performed Focused Ion Beam Scanning Electron Microscopy (FIB-SEM) on stage 2 SGs. We acquired a 3D volume of a single granule with an isotropic resolution of 6 $\mathrm{nm}$ (Fig. 2a), and used a cropped region (160 x 334 x 200, xyz) within the granule in different orientations for segmentation and 3D reconstruction (Fig. $2 \mathrm{~b}$ and $2 \mathrm{c}-2 \mathrm{f}$ ). Semi-automated segmentation was performed and for the purpose of illustration, the discs are pseudocolored in red and the electron-dense matrix in green 
(Fig. 2b'). Manual analysis of the 3D reconstruction volume reveals that the electron-dense matrix has indentations (arrows in Fig. 2g) into which the discs are embedded (Fig. 2g'-g''). The discs were found to be embedded at the peripheral surface of the electron-dense matrix when viewed at different rotational angles (Fig. 2h-j) and not within the electron-dense matrix as revealed by the orthogonal sections through the 3D volume (Fig. 2k). Therefore, our data suggest that the discs are present around the periphery of the electron-dense matrix. Taken together, our data indicate each mature secretory granule contains multiple distinct structures with defined dimensions and spatial arrangements with respect to each other.

\section{Secretory cargo undergoes regulated restructuring during secretory granule maturation}

To understand the development of the distinct structures present within mature secretory granules, we imaged secretory granules beginning at early stages of granule biosynthesis (Stage 1) using TEM. During stage 1, the SG displays a gradient of secretory granules at various stages of maturation, with granule biogenesis beginning at the distal tip and proceeding proximally (Fig. 1b). We therefore sliced the SGs from proximal to distal (at $25 \mu \mathrm{m}$ intervals) to capture secretory granules at various stages of development. In the initial proximal sections captured, we observed several small granules scattered throughout the cytoplasm ranging between 200-400 $\mathrm{nm}$ in diameter (Fig. 3a and Sup.Fig. 1c). These small granules often had no discernable internal structures or the beginnings of what appear to be irregularly-spaced filaments (Fig. 3a'). In subsequent sections, we observed granules ranging from 600-900 $\mathrm{nm}$ in diameter that contained more electron-dense filaments and a few small regions with higher-electron density (Fig. 3b-b' and Sup.Fig. 1d). Further distal sections revealed larger diameter granules (900-1200 nm) with more distinct filaments in addition to larger electron-dense regions (Fig. 3c-c' and Sup.Fig. 1e). In sections with secretory granules ranging from 1200-1800 nm in diameter, filaments are now very distinct and arranged in evenlyspaced, parallel stacks throughout the granule (Fig. 3d-3d'). Additionally, very distinct and large electrondense regions are present in these granules (Fig. 3c-c' and Sup.Fig. 1f).

We next analyzed stage 2 SGs, where most of the granules observed had diameters greater than $1800 \mathrm{~nm}$ (Fig. 3e-e' and Sup.Fig. 1g). During this stage, each secretory granule has filaments arranged in evenly spaced parallel stacks. We also observed the discs that line the periphery of the distinct and large electron-dense matrix at this stage. Taken together, these results suggest that secretory cargo undergoes regulated restructuring during secretory granule maturation, with filaments forming first, followed by electron-dense aggregates and then finally discs that form along the periphery of the electron-dense regions. Moreover, our results further suggest the existence of multiple unique cargo proteins in each granule that adopt distinct structures during secretory granule maturation. 


\section{Distinct mucins form unique intragranular structures in vivo}

To determine the nature of the unique intragranular structures observed in mature secretory granules in vivo, we next performed RNAi-mediated knockdown of the genes encoding the major secretory proteins/mucins known to be expressed in the SGs, $s g s l$ and $s g s 3^{28,29,30,44,45,46}$ ). RNAi-mediated knockdown of $\operatorname{sgSl}\left(s g S 1^{\text {TRIP.HMCO2393 }}\right.$ ) resulted in a complete loss of the disc structures typically seen in mature $w t$ granules (Fig. $4 \mathrm{a}$ vs $4 \mathrm{~b}$ ). However, no discernable changes in the organization/arrangement of the filaments or the electron-dense matrix was observed (Fig. 4a vs. 4b). QPCR, protein gels and western blotting confirmed that RNAi to $s g s 1$ resulted in a $>95 \%$ reduction in $s g s 1$ gene expression (Sup.Fig. 2a) and the specific loss of the O-glycosylated protein band previously identified as Sgs1 ${ }^{47}$ (Fig. 4d and 4e). Taken together, these results suggest that Sgs1 forms the discs structures.

We next performed RNAi-mediated knockdown of $\operatorname{sgs} 3$ ( $\operatorname{sgs}^{\text {TRIP.HMJ3002I }}$ ), the most abundant mucin present in the SGs. Upon performing TEM on $\operatorname{sgs} 3^{\text {TRiP.HMJ30021 }}$ SGs, we found complete loss of the filament bundles and the electron-dense dots (that likely represented cross sections of the filament bundles) (Fig. 4a vs. 4c). Additionally, we also observed that the $s g s 3^{\text {TRIP.HMJ30021 }}$ secretory granules were smaller in diameter than $w t$ controls, likely due to the loss of the filaments which occupy more than $50 \%$ of the granule volume (Fig. 4a vs. 4c). While the filament bundles were absent, the discs and the electron-dense matrix were still present in the $\operatorname{sgs}^{\text {TRIP.HMJ30021 }}$ secretory granules. QPCR and western blotting demonstrated that RNAi to sgs 3 resulted in $>95 \%$ reduction in $s g s 3$ gene expression (Sup.Fig. $2 \mathrm{~b}$ ) and loss of the Sgs 3 protein band (Fig. 4d and 4f), indicating specificity of this knockdown and further supporting the conclusion that the Sgs 3 mucin adopts a bundled filament structure within a mature secretory granule. Taken together, our results indicate that 2 distinct mucins undergo unique packaging events within the same secretory granule to form distinct intragranular structures upon granule maturation.

\section{Intragranular restructuring is dependent on the v-ATPase proton pump}

We next set out to interrogate the factors that drive secretory granule maturation and the restructuring of secretory cargo. Previous in vitro studies have suggested that an acidic $\mathrm{pH}$ is required for conformational changes necessary to package large proteins, such as mucins, into more compact structures within secretory granules ${ }^{16,48}$. To test this hypothesis in vivo, we examined the expression of genes that encode subunits of the v-ATPase proton pump in SGs at various stages of secretory granule development (Sup.Fig. 2c). vATPases are a large family of multi-subunit protons pumps, composed of two functional domains V1 and $\mathrm{V} 0$, that are responsible for regulation of the $\mathrm{pH}$ in intracellular compartments ${ }^{49}$. Interestingly, we found that one gene encoding the v-ATPase 16kD subunit-1 (vhal6-1) was highly expressed during all stages of secretory granule development and peaked in expression during stage 2 (Sup.Fig. 2c). We next tested the effect of this gene on intragranular morphology by performing RNAi in SGs using 2 independent lines 
(VDRC\# 49291 and VDRC\# 104490). Each line (under the control of the c135-Gal4 driver) showed a greater than 75\% knockdown of vha16-1 transcripts in the SGs (Sup. Fig. 2e). Confocal imaging of stage 2

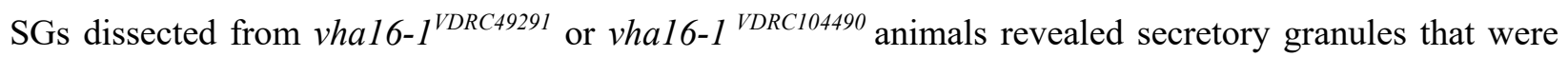
circular and swollen in appearance when compared to $w t$ granules (Fig. 5a, 5b and 5c). Quantitation of circularity shows statistically significant differences between $w t$ and vha16-1 VDRC49291 or vha16-1 VDRC104490 secretory granules (Fig. 5f). Additionally, treatment of $w t$ SGs with the v-ATPase inhibitor Bafilomycin$\mathrm{A} 1{ }^{50}$ ) resulted in swollen and circular secretory granules (Fig. $5 \mathrm{~d}$ and e), further supporting a role for vATPase activity and $\mathrm{pH}$ in secretory granule morphology.

To further understand the ultrastructural changes resulting from the loss of the v-ATPase, we next performed TEM on vha16-1 $1^{\text {VDRC49291 }}$ SGs. Interestingly, we saw significant changes in intragranular morphology in the absence of vha16-1. vha16-1 $1^{\text {VDRC49291 }}$ SGs lacked the distinct Sgs3 filament bundles, suggesting that an acidic $\mathrm{pH}$ is required for the formation of these structures (Fig. $5 \mathrm{~g}$ and $5 \mathrm{~h}$ ). The Sgs1 discs and the electron-dense matrix were still present but were confined to the periphery of the secretory granules, suggesting that defective packaging of Sgs3 may disrupt the spatial distribution of other proteins. These results suggest that vha16-1 and the acidic $\mathrm{pH}$ it induces are required for the appropriate folding/restructuring of $\mathrm{Sgs} 3$ in vivo.

\section{Loss of chloride ion channel proteins disrupt restructuring and intragranular morphology}

Along with acidic $\mathrm{pH}$, in vitro studies have suggested a role for various ions in charge shielding to promote packaging of mucin polymers ${ }^{16,51,52}$. QPCR data from SGs at various stages revealed that one calcium channel (fwe) and two chloride channels (Clic and $\mathrm{ClC}-\mathrm{c}$ ) are highly expressed and upregulated during stages 1 and 2 (Sup.Fig. 2d). We therefore examined the effects of these ion channels on secretory granule morphology and cargo restructuring by performing RNAi to each. Clic, which encodes an intracellular chloride transporter localized to the mitochondria ${ }^{53}$, was knocked-down using 2 independent lines (VDRC \#105975 and VDRC \#28303). RNAi to Clic using either line (under the control of the c135-Gal4 driver) gave in a greater than $75 \%$ decrease in gene expression by QPCR (Sup.Fig. 2f) and resulted in granules that were circular, swollen and much larger in diameter than $w t$ when observed using confocal microscopy (Fig. 6a-6c). TEM examination of stage 2 Clic $^{V D R C 105975}$ SGs revealed a notable loss of the electron-dense matrix relative to $w t$ (Fig. $6 \mathrm{f}$ vs. 6g). Likewise, the Sgs1 discs, which are normally associated with the electrondense matrix, were found to be scattered throughout the granule. Sgs3 filament bundles could still be seen but were sparse relative to $w t$ (Fig. $6 \mathrm{f} \mathrm{vs.} 6 \mathrm{~g}$ ). Along with the disrupted intragranular morphology, we also saw swollen and damaged mitochondria (Sup. Fig. 3a-3d), suggesting that defects in intragranular morphology could also be the result of defective mitochondrial function. 
We next examined the role of second most abundantly expressed chloride channel $(\mathrm{ClC}-\mathrm{c})$ (Sup.Fig. 2d) in intragranular morphology. $C l C-c$ is a homolog of human $C L C N 3(C L C-3)$ which encodes a voltage-gated chloride transporter that is involved in endosomal acidification by functioning as a proton antiporter ${ }^{54,55}$. RNAi to $\mathrm{ClC}$ - $c$ was performed using two independent lines (VDRC \#6466 and VDRC \#106844) (Sup. Fig. 2g) and resulted in granules that were grossly misshapen when examined using confocal microscopy (Fig. 6a vs. 6d-6e). ClC-c $c^{V D R C 6466}$ and $C l C-c^{V D R C 106844}$ secretory granules were oblong compared to the oval granules seen in $w t$ SGs. TEM analysis of $C l C-c^{V D R C 6466}$ revealed major changes in intragranular structures, with the electron-dense matrix fragmented into smaller islands and scattered throughout the granule lumen (Fig. 6f vs. 6h). The Sgs1 discs were still present around the periphery of the electron-dense matrix but were much less distinct in structure. The Sgs3 filaments maintained their parallel stacked arrangement and did not show any abnormal organization. In all the TEM sections observed we did not find any evidence of damaged mitochondria as was seen upon the loss of Clic in the SGs. These results suggest that the loss of $C l C-c$ is specifically affecting the restructuring of the electron-dense matrix and the organization of Sgs1.

\section{Loss of the calcium channel fwe disrupts secretory granule maturation}

To determine how calcium influences intragranular morphology, we analyzed the gene flower (fwe), which encodes a transmembrane calcium ion channel that regulates synaptic vesicle exo- and endocytosis ${ }^{56}$. Indeed confocal imaging of stage 2 SGs expressing a fluorescently tagged fwe genomic rescue construct (Fwe-YFP) ${ }^{56}$ shows that Fwe localizes to secretory granule membranes (Fig. 7a). RNAi knockdown of $f w e$ was performed using two independent RNAi lines (VDRC \#39596 and TRiP.GL01498) (Sup. Fig. 2h).

Confocal imaging of stage $2 f w e^{V D R C 39596}$ and $f w e^{\text {TRiP.GLO1498 }}$ SGs showed regions containing very large granules with low-GFP intensity (Fig. 7b vs.7d, e; white arrowheads). TEM analysis of SGs from stage 2 fwe $e^{V D R C 39596}$ animals showed large granules that lacked the Sgs 1 discs and the electron-dense matrix (Fig. 7e vs. 7f). Additionally, the organized bundled filaments typical of Sgs3 were no longer present. Instead, secretory granules were filled with very long parallel filaments that spanned the entire lumen of the granule, making it look like a ball of yarn (Fig. 7e vs. 7f). Therefore, loss of fwe disrupts the restructuring of Sgs1 and Sgs3, suggesting that the formation of these structures is calcium dependent in vivo. 


\section{Cargo glycosylation affects specific intragranular structures and secretory granule morphology}

Previous studies from our group have shown that loss of an O-glycosyltransferase (PGANT9B) results in secretory granules that are abnormal in morphology and no longer circular/oval ${ }^{47}$. This study identified the cargo protein Sgs3 as a substrate of PGANT9B. To further examine how loss of PGANT9B affects secretory granule morphology, we examined pgant 9 mutants ( $\operatorname{pgant}^{4} / \mathrm{Df}(2 \mathrm{R})$ ) by TEM. Loss of pgant 9 results in secretory granules that are abnormal in shape relative to $w t$ (Fig. 8a-8d). Like $w t$, pgant $9^{4} / D f(2 R)$ granules contained the electron-dense matrix, the Sgs1 discs and the Sgs3 filament bundles. However in pgant 9 mutants, the Sgs1 discs were less pronounced and their positioning along the electron-dense matrix is notably different that $w t$. Additionally, the spacing between the Sgs3 filaments was significantly reduced (Fig. 8e-8g), suggesting that O-glycosylation of Sgs3 may regulate the distance between adjacent filaments. We hypothesize that the irregular granule shapes may be due to the less mobile nature of the more tightly packed Sgs3 filaments. Indeed, examination of several TEM micrographs of $\operatorname{pgant} 9^{4} / D f(2 R)$ granules suggests that the mixing of granule contents may be constrained upon homotypic fusion of granules. These observations are in line with our previous study that showed that abnormal granule morphology became evident later during granule maturation as homotypic fusion was occurring ${ }^{47}$. Taken together, we demonstrate that multiple distinct proteins undergo ordered restructuring during secretory granule maturation that is dependent on granule acidification, ion changes and cargo glycosylation. These restructuring events allow for intragranular spatial segregation of distinct cargo. 


\section{DISCUSSION}

Here we show that individual secretory granules contain multiple distinct proteins that undergo temporally-ordered restructuring during secretory granule maturation to adopt distinct intragranular structures. Moreover, we further characterize the identity of each structurally-distinct protein and the factors responsible for each developing their unique structure in mature granules. Our results support a model whereby multiple cargo proteins destined to be a part of the secretate are packaged into the same secretory granule but are segregated via orchestrated restructuring during secretory granule maturation. Indeed, these results support our prior studies in the SG that show that the cargo receptor Tangol acts to form a functional docking site between the ER and Golgi that would allow protein passage between these compartments, rather than selective protein partitioning ${ }^{36}$. The packaging of multiple proteins into a single immature granule obviates the need for specific sorting receptors for individual proteins, and instead relies on segregation via intragranular compartmentalization. Our results highlight how secretory cells under a high secretory burden may rely on intragranular rather than intergranular segregation of distinct sercretory cargo.

Through a combination of genetics and ultrastructural analysis of secretory granules at different stages of development, we identify two of the unique structures as being those of the major secretory mucins, Sgs 1 and Sgs $3{ }^{57}$. Previous studies using a Sgs3-GFP reporter have shown that immature granules grow in size and mature by undergoing homotypic fusion ${ }^{36,38,39,40}$. Maturation of immature secretory granules by homotypic fusion has also been reported in endocrine and neuroendocrine cells ${ }^{58,59}$. When examining nascent granules, at different developmental stages, we did not find any containing exclusively Sgs1 or Sgs3 structures. Rather, the intragranular structures formed by these two mucins became evident gradually over time as the granules matured, suggesting that multiple mucins are packaged into a single secretory granule and that each undergoes regulated restructuring during secretory granule maturation to adopt a distinct intragranular structure. Interestingly, each mucin adopted a structure reminiscent of structures seen in in vitro reconstitution experiments performed with segments of mammalian mucins. Sgs1 adopted a disc-like structure, similar to that seen upon reconstitution of a portion of MUC5B in vitro, where head-to-head N-terminal stacking that is proposed to account for this structure ${ }^{25,26,27}$. Likewise, Sgs3 restructured into parallel filament bundles, similar to those seen upon in vitro reconstitution of portions of MUC2 ${ }^{16,24}$. The importance of the packaging of MUC2 is evident when one considers tha this mucin, like many mucins that line and protect epithelia, must undergo regulated expansion into a net-like structure that lines the intestinal environment, conferring protection from microbial and mechanical damage ${ }^{21}$. Similar to mammalian mucins, Drosophila mucins also form a lining that mediates attachment to a substrate to 
enable metamorphosis, suggesting that their packaging and expansion upon secretion must also be similarly regulated.

We have further identified the factors that mediate the ordered restructuring of the Drosophila mucins during secretory granule maturation in vivo. The intermediate steps of secretory granule formation/granule maturation after export from the trans-Golgi are poorly understood for mammalian mucins, although roles for calcium ions and $\mathrm{pH}$ have been shown to be important for this process in vitro 48, 52. Indeed, we find that two genes controlling $\mathrm{pH}$ and calcium concentration (vhal6-1 and fwe, respectively) are differentially regulated during secretory granule maturation and are essential for mucin restructuring in vivo. Loss of vhal6-1, which encodes a subunit of the vATPase proton pump, disrupted the restructuring of Sgs3 and the association of Sgs1 discs with the electron-dense matrix. Leading to grossly abnormal secretory granules and highlighting a crucial role for $\mathrm{pH}$ changes in the restructuring/segregation of cargo proteins in vivo. Loss of fwe, which encodes a calcium channel present on the granular membrane, resulted in the complete disruption of all intragranular structures, highlighting the crucial importance of calcium in the formation of all structures and the spatial segregation of all cargo. Previous work has demonstrated that the acidic $\mathrm{pH}$ and high calcium concentrations at the trans-Golgi can induce aggregation of proteins and their selective sorting into secretory granules ${ }^{60,61}$. Likewise, proinsulin processing to produce mature insulin requires cleavage by endoproteases that are active in the low $\mathrm{pH}$ and high calcium ion environment of the trans-Golgi and in immature secretory granules ${ }^{62,63}$. With regard to mucins, a pH-dependent autocatalytic cleavage in the C-terminal cysteine-rich VWD domain of MUC2 and MUC5AC has been reported to occur in the trans-Golgi network ${ }^{64,65}$. Biophysical and single particle EM analysis proposed that the MUC2 mucin is packaged as concatenated polygonal rings, with the mucindomains projecting perpendicular from the base of the rings as parallel rods in a calcium and $\mathrm{pH}$-dependent manner within secretory granules ${ }^{16}$. However, an alternative model suggests that MUC2 complexes exist as linear polymers ${ }^{24}$ that are also calcium and $\mathrm{pH}$ dependent. Previous studies utilizing single particle EM analysis also identified calcium-mediated cross-links in the VWD-3 domain of MUC5B that could mediate stacking and condensation of the mucin into a round disc-like structure around a central node ${ }^{25,26,27}$. Taken together, our in vivo studies support a crucial role for $\mathrm{pH}$ and calcium in intragranular packaging of endogenous mucins and are well-aligned with previous reconstitution/folding studies that have highlighted a role for calcium and $\mathrm{pH}$ in the restructuring of mucins in vitro $16,25,26,27$.

In addition to genes controlling $\mathrm{pH}$ and calcium, we found that two chloride channel proteins (Clic and $\mathrm{ClC}-\mathrm{c})$ were also upregulated during secretory granule maturation and had effects on cargo morphology. The CLIC ( $\mathrm{Cl}^{-}$intracellular channel) proteins are a highly conserved family of dimorphic proteins that exist in soluble and membrane forms ${ }^{66}$. CLICs are multifunctional proteins that have been reported to play roles in apoptosis ${ }^{67}$, membrane trafficking ${ }^{68}$, tubulogenesis ${ }^{69}$ and cell differentiation ${ }^{70}$, 
and display differential localization ${ }^{71}$ on nuclear membranes ${ }^{72}$, the ER ${ }^{73}$, mitochondria ${ }^{53}$ and secretory vesicles of hippocampal neurons ${ }^{74}$. Drosophila encodes a single homolog of CLIC ${ }^{75}$ and knockdown of Clic in SGs resulted in swollen secretory granules with disrupted intragranular morphology and damaged mitochondria. The round and swollen granules with disorganized intragranular morphology seen upon knockdown of Clic were reminiscent of the phenotype seen upon the loss of vhal6-1. Since $\mathrm{Cl}^{-}$is considered to provide an electrical shunt for the acidification of most organelles of the secretory and endocytic pathway, it is conceivable that Clic might be affecting intragranular morphology by indirectly regulating the $\mathrm{pH}$. The other chloride channel upregulated during secretory granule maturation, $\mathrm{ClC}-\mathrm{c}$, is a homolog of the human CLC-3, which functions as an electrogenic $2 \mathrm{Cl}^{-} / \mathrm{H}^{+}$exchanger ${ }^{54}$, assists in acidification of endosomes by supplying countercurrents for the vesicular $\mathrm{H}^{+}$-ATPase ${ }^{76}$, and also been proposed to be involved in vesicular $\mathrm{Cl}^{-}$accumulation and ion homeostasis ${ }^{77}$. In our $C l C-c$ knockdown, we find a severe defect in the shape of the granule, fragmentation of the electron-dense matrix and mislocalization of Sgs1 discs. Loss of $\mathrm{ClC}-\mathrm{c}$ may impair $\mathrm{Cl}^{-}$accumulation, leading to alterations in $\mathrm{pH}$ of the granule during the maturation steps. However, the loss of $\mathrm{ClC}-\mathrm{c}$ results in a distinct phenotype from those seen upon loss of vha16-1 or Clic, suggesting a unique role for this ion channel.

Finally, we demonstrate how O-glycosylation, a protein modification abundant on mucins ${ }^{78,79}$, influences the structures of the mucins, thereby influencing secretory granule morphology. Previous studies from our group demonstrated that loss of one glycosyltransferase (pgant 9 ) resulted in secretory granules that displayed irregular morphologies ${ }^{47}$. In the present study, ultrastructural analysis demonstrated that the loss of pgant 9 results in Sgs3 filaments that are closer together. Given that the abnormal secretory granule morphology is most evident once granules begin to undergo homotypic fusion and mature, we hypothesize that the irregular granule shapes may be due to the less mobile nature of the more tightly packed Sgs3 filaments.

In summary, we demonstrate that multiple proteins undergo regulated restructuring during organized secretory granule maturation to form unique structures and that these restructuring events are both $\mathrm{pH}$ and ion dependent. We present a new model for regulated intragranular cargo segregation to ensure efficient storage, secretion and unfolding of large extracellular proteins such as mucins that function in the protection of epithelial surfaces. 


\section{METHODS}

\section{Fly strains}

All fly strains and crosses were raised and maintained on MM fly media (KD Medical, Inc.), the ambient conditions were set to a $12: 12$ hours of dark and light cycle at $25^{\circ} \mathrm{C}$. The following stocks were obtained from the Bloomington Drosophila stock center, c135-Gal4 (BL6978, $\left.w^{1118} ; P\left\{w^{+m W \cdot h s}=G a w B\right\} c 135\right)$, sgs3GFP (BL5885, w[*]; $\left.P\left\{w\left[+m^{*}\right]=s g s 3-G F P\right\} 3\right), \quad \operatorname{sgs} 1-R N A i \quad$ (BL51421, $y[1] \quad v[1] ; \quad P\{y[+t 7.7]$ $v[+t 1.8]=T R i P . H M C 02393\} a t t P 2), \quad s g s 3-R N A i \quad$ (BL62944, $\quad y[1] \quad v[1] ; \quad P\{y[+t 7.7]$ $v[+t 1.8]=T R i P . H M J 30021\} a t t P 40), \quad f w e-Y F P \quad(B L 51615, \quad w[*] ; \quad P\{w[+m C]=f w e-8.2 g-Y F P-G F P-$ RFP 22-1-2), fwe-RNAi (BL43157, y[1] v[1]; P\{y[+t7.7] v[+t1.8]=TRiP.GL01498\}attP40), pgant9 deficiency line (BL7546, Df(2R)Exel6064), pgant $9^{4}{ }^{47}$ ). RNAi stocks expressing hairpin double stranded RNA was obtained from the Vienna Drosophila RNAi Center (VDRC), stocks used are VDRC\#60000 $\left(w^{1118}\right)$, VDRC\#49291 and VDRC\#104490 for CG3161 (vha16-1), VDRC\#6466 and VDRC\#106844 for CG5284 (ClC-c), VDRC\#105975 and VDRC28303 for CG10997 (Clic) and VDRC\#39596 for CG6151 (fwe). The c135-Gal4 driver stock was recombined with sgs3-GFP to generate c135-Gal4, sgs3-GFP to visualize granules and induce RNAi in SGs. c135-Gal4, sgs3-GFP crossed to VDRC\#60000 ( $\left.w^{1118}\right)$ was used a control for the RNAi experiments.

\section{Transmission electron microscopy}

SGs were dissected in ice cold Schneider's medium and fixed in 4\% formaldehyde/ $2 \%$ glutaraldehyde $/ 0.1 \mathrm{M}$ sodium cacodylate buffer $(\mathrm{pH} 7.4)$ overnight at $4{ }^{\circ} \mathrm{C}$. After overnight fixation salivary glands were postfixed in $1 \%$ osmium tetroxide for 1 hour on ice and overnight in $1 \%$ uranyl acetate at $4{ }^{\circ} \mathrm{C}$. The following day SGs were dehydrated in graded ethanol series followed by propylene oxide and gradually infiltrated with increasing ratio of EMbed 812 resin (EMS, \#14120) to propylene oxide with final infiltration in $100 \%$ resin. Next, SGs were embedded in Beem capsules (EMS, \#70000, Size 00) and polymerized in the oven at $60 \mathrm{C}$ for 48 hours. Ultrathin sections $(70 \mathrm{~nm}$ ) were cut on ultramicrotome (Leica EM UT7) and Digital micrographs were acquired on JOEL JEM 1200 EXII operating at 80Kv and equipped with AMT XR-60 digital camera. For High-pressure freezing and free substitution, SGs were dissected in Schneider's medium and transferred to a Brass disc (type-A planchet, cavity depth $0.2 \mathrm{~mm}$ ). Excess of Schneider's medium was carefully removed and immediately $10 \mu 1$ of $20 \%$ BSA was pipetted and uniformly distributed. After inspecting sample orientation another Brass disc (Type-B planchet) was placed on top with flat surface down to seal the assembly. The assembled specimen chamber was frozen using a highpressure freezing system (Leica EM ICE). The frozen sample were transferred to cryovials in liquid nitrogen vapor and transferred to pre-cooled $\left(-90^{\circ} \mathrm{C}\right)$ freeze substitution unit (Leica EM AFS). Freeze 
substitution was performed using a mixture of $0.12 \%$ aqueous Uranyl acetate in anhydrous acetone using the following program: $-90^{\circ} \mathrm{C}$ for $44 \mathrm{hrs}$ followed by slow warming from $-90^{\circ} \mathrm{C}$ to $-47^{\circ} \mathrm{C}\left(15^{\circ} \mathrm{C} / \mathrm{hr}\right)$ and at $-47^{\circ} \mathrm{C}$ freeze substitution solution was removed and samples were washed 3 times for 10 mins with acetone. Resin infiltration was performed by incubating samples in increasing concentrations of Lowicryl HM20 resin $(25 \%, 50 \%, 75 \%)$ in acetone with final 3 incubations with $100 \%$ resin lasting for $48 \mathrm{hrs}$. The samples were gradually warmed from $-47^{\circ} \mathrm{C}$ to $24^{\circ} \mathrm{C}\left(5^{\circ} \mathrm{C} / \mathrm{hr}\right)$ and polymerized under UV over a period of $48 \mathrm{hrs}$. Ultrathin sections (70nm) were cut on ultramicrotome (Leica EM UT7) and postained with 2\% of uranyl acetate for 10 mins and lead citrate for 2 mins. Digital micrographs were acquired on JOEL JEM 1200 EXII operating at $80 \mathrm{kV}$ and equipped with AMT XR-60 digital camera.

\section{FIB-SEM imaging and segmentation}

FIB-SEM imaging was performed as described in ${ }^{80}$, the acquired dataset of a single granule was cropped $(160 \times 334 \times 200, x y z)$ to include electron-dense matrix and discs and used further for segmentation. Image processing was peformed using Fiji (NIH, ImageJ.net), for the initial image processing, variable contrast was adjusted using the Enhance Local Contrast (CLAHE) plugin and a Gaussian blur 3D ( $\boldsymbol{\sigma}=2)$ was applied to the image stack. Feature segmentation (electron-dense matrix and discs) was performed using the Trainable Weka segmentation plugin (version 3.2.34), three classifiers were trained to include electrondense matrix, discs and the filaments using the FastRandomForest classifier. The resulting probability maps after training were manually inspected and the classifiers were retrained to minimize feature overlap. After refinement of the model, probability maps were exported as 32-bit hyperstack. 3D visualization, pseudocoloring and volume rendering of probability maps of the individual features was performed using Imaris (version 9.3.0, Bitplane.com).

\section{Western blotting}

SGs were dissected from third instar larva and squished in 50 $\mu 1$ RIPA buffer (Sigma) supplemented with $1 X$ Halt Protease Inhibitor (Thermo Scientific). Protein extracts were loaded on to NuPAGE 4-12\% BisTris gel (Invitrogen). For Coomassie blue staining, the gel was rinsed with ultrapure water three times and stained with SimplyBlue Safestain (Thermo Scientific). For western blotting, proteins were transferred from gel onto nitrocellulose membranes. The membranes were blocked with Odyssey Blocking Buffer (Li-COR) and incubated with IRDye 800CW-conjugated PNA (1:5000) and the anti-tubulin antibody (1:1000, Cell Signaling Technology) or anti-Sgs3 antibody (1:2000) overnight at $4{ }^{\circ} \mathrm{C}$. After washing with PBS containing $0.1 \%$ Tween-20 (PBST), the membrane was incubated with IRDye 680LT-conjugated antirabbit $\operatorname{IgG}(1: 10000, \mathrm{Li}-\mathrm{COR})$ for $1 \mathrm{hr}$ at room temperature then washed with PBST and PBS, then scanned by a Li-COR Odyssey Infrared Imaging System. 


\section{Real-time PCR}

SGs were dissected in Schneider's medium and 10 pairs of SGs were pooled in DNA/RNA shield. RNA was extracted using the Direct-zol RNA Microprep kit (ZymoResearch) following the manufactures instructions and cDNA was synthesized from $1 \mu \mathrm{g}$ of total RNA using iScript cDNA Synthesis Kit (BioRad). qPCR was performed on a CFX96 real time PCR thermocycler (Bio-Rad) using the SYBR-Green PCR Master Mix (Bio-Rad). Primers used for qPCR are listed in Sup.Table-1.

\section{Morphometric analysis}

ImageJ was used to perform morphometric analysis on the acquired TEM micrographs. Briefly, micrographs were calibrated, and the inter-filaments distance and the disc diameter were measured using the straight line and oval selection tools. A total of 100 measurements were performed across 10 micrographs and GraphPad Prism was used to perform statistical analysis. Student $t$-test was used to compare the inter-filaments distance between wild-type and pgant 9 mutants and significant differences are reported for $\mathrm{p}$-values $<0.01$. Circularity measurements of the secretory granules was performed as previously described ${ }^{47}$

\section{SGs imaging, Lysotracker and V-ATPase inhibitor treatment}

SGs were imaged as previously dercribed in ${ }^{35}$. SGs were staged according to sgs3-GFP ${ }^{33}$ reporter and non-secreting glands were dissected in Schneider's medium (Thermo Scientific) and transferred to glass bottom culture dishes (MatTek). A polycarbonate membrane filter (Sterlitech) was gently placed on the top of the glands and the samples were imaged on Nikon A1R+ confocal microscope equipped with a PlanApochromat IR 60x/1.27 NA water immersion objective (For LysoTracker or V-ATPase inhibitor treatment, SGs were dissected in Schneider's medium and incubated with Bafilomycin-A (500 nM) or LysoTracker $(1 \mu \mathrm{M})$ in Schneider's medium for 1 hour at room temperature. After incubation, SGs were rinsed with Schneider's medium and transferred to glass bottom culture dishes (MatTek) and imaged as described above. Acquired images were processed in Adobe Photoshop CC and compiled into panel using Adobe Illustrator CC 2019.

\section{Data availability}

Data supporting the findings of this manuscript are available from the corresponding author upon reasonable request. 


\section{Acknowledgements}

We would like to thank our colleagues for many helpful discussions. We also thank the Bloomington Stock Center, the Developmental Studies Hybridoma Bank and the Vienna Drosophila RNAi Center for providing fly stocks, antibodies and other reagents. This research was supported by the Intramural Research Program of the NIDCR at the National Institutes of Health (Z01-DE-000713 to K.G.T.H.) and the NIDCR Imaging Core (ZIC DE000750-01).

\section{Author contributions}

Z.A.S., L. Z., D. T. T. and K.G.T.H. designed and planned the research. Z.A.S. and L. Z. performed the experiments. Z. A.S., L. Z., D.T.T, C.K.E.B, and K.G.T.H. analyzed and discussed the data. Z.A.S. and K.G.T.H. wrote the paper.

\section{Competing interests}

The authors declare no competing interests. 


\section{FIGURE LEGENDS}

\section{Figure 1. Mature secretory granules have distinct intragranular structures}

Illustrations representing different stages of the third instar larval salivary glands (SGs) based on the expression pattern of Sgs3-GFP at stage 0 (a), stage 1 (b), stage 2 (c) and stage 3 (d). Higher magnification confocal image of secretory granules at stage 2 shows their round/oval shape (e). Transmission electron micrographs (TEM) of a stage 2 SG showing a single mature secretory granule with distinct intragranular structures (f). Magnified views of the boxed regions show that the mature granule has at least three distinguishable structures: electron-dense filaments arranged in parallel bundles ( $\mathbf{g}$ and $\mathbf{h}$ ), electron-lucent discs (i; black arrowhead) and an electron-dense matrix (i; white arrowhead). In most of the stage 2 granules observed under TEM, the electron-lucent discs were closely associated with the electron-dense matrix (i; white arrowhead). Morphometric analysis of the stage 2 granules showing the inter-filament distances and the average diameter of the discs (j). Scale bars, $5 \mu \mathrm{m}$ for $\mathbf{e}, 500 \mathrm{~nm}$ for $\mathbf{f}$ and $100 \mathrm{~nm}$ for $\mathbf{g}$-i. Representative images from three independent experiments are shown.

\section{Figure 2. Unique associations between distinct intragranular structures}

Focused ion beam scanning electron microscopy (FIB-SEM) of stage 2 secretory granules (a-f) shows slices through the region encompassing the discs and electron-dense matrix. Pseudo-colored discs (red) and electron-dense matrix (green) (b') were reconstructed in 3 dimensions in $\mathbf{g}$-k. White arrows in $\mathbf{g}$ highlight indented regions of the electron-dense matrix in which the discs are embedded. Images are rotated $90^{\circ}(\mathbf{h})$, $180^{\circ}$ (i) and $270^{\circ}(\mathbf{j})$. Orthogonal slice showing only the red pseudicolored discs is shown in $\mathbf{k}$. Scale bars, $600 \mathrm{~nm}$ for $\mathbf{a}$ and $100 \mathrm{~nm}$ for $\mathbf{b}-\mathbf{k}$.

\section{Figure 3. Secretory granule cargo undergoes regulated restructuring}

Transmission electron micrographs (TEMs) of stage 1 and stage 2 SGs show the formation of distinct structures over time during secretory granule maturation. Early stage 1 secretory granules with diameters ranging from 200-400 $\mathrm{nm}$ show the first appearance of irregularly spaced electron-dense filaments (a-a'). As granules reach 600-900 nm in diameter (b-b'), the electron-dense matrix starts to appear. In the granules with diameters ranging from 900-1200 nm (c-c'), electron-lucent discs become visible along with filament bundles and electron-dense regions. Secretory granules that are 1200-1800 nm in diameter (d-d') have large areas of electron-dense material and the filaments are more evenly spaced. Granules greater than $1800 \mathrm{~nm}$ in diameter (e-e') display ordered filament bundles and distinct electron-lucent discs in close association with the electron-dense regions, as is seen in fully mature granules. Scale bars, $200 \mathrm{~nm}$ for a, $600 \mathrm{~nm}$ for b-e, and $100 \mathrm{~nm}$ for a'-e' . Representative images from three independent experiments are shown. 
Figure 4. Distinct mucins form unique intragranular structures within secretory granules

TEMs on $w t$ secretory granules (a) were compared to those in which RNA interference (RNAi) was performed to genes encoding 2 distinct mucins, sgs1 (b) and $\operatorname{sgs} 3$ (c). RNAi to $\operatorname{sgs} 1$ ( $\operatorname{cgs} 1^{\text {TRiP.HMC02393) }}$ results in the loss of the electron-lucent discs (b), indicating that Sgs1 forms the disc structures. RNAi to $\operatorname{sgS} 3\left(\operatorname{sgs} 3^{\text {TRIP.HMJ3002I }}\right.$ ) results in the loss of the filament structures that occupy most of the granule (c) and results in secretory granules that are much smaller in size than $w t$. Loss of the Sgs1 or Sgs3 proteins upon sgs $1^{\text {TRIP.HMC02393 }}$ or $\operatorname{sgs} 3^{\text {TRIP.HMJ3002I }}$ RNAi, respectively, was confirmed by SDS-PAGE and coomassie blue staining (d; red arrows). Western blots probed with the lectin peanut agglutinin (PNA), which detects the glycosylated mucins, also show the specific loss of the Sgs1 band (e; red arrow) and Sgs3 (f; red arrows). Scale bars, $600 \mathrm{~nm}$ for a-c. Representative images from three independent experiments are shown..

\section{Figure 5. Intragranular restructuring is dependent on the v-ATPase proton pump}

Confocal images of $w t$ (a) and RNAi-mediated knockdown of the vhal6.1 subunit with two independent RNAi lines, vha16.1 ${ }^{\text {VDRC49291 }}$ (b) and vha16.1 VDRC104490 (c) are shown. Circularity measurements for each genotype are graphed in f. SGs untreated (d) or incubated with the v-ATPase inhibitor Bafilomycin A1 (e) recapitulate the round secretory granule phenotype seen upon knockdown of vhal6.1. TEMs of stage $2 w t$ (g) and vha16.1 ${ }^{\text {VDRC49291 }}$ (h) SGs showing the effects of vhal6.1 knockdown on intragranular structures, including the loss of the Sgs1 discs and the Sgs3 filaments. Scale bars, $10 \mu \mathrm{m}$ for $\mathbf{a}-\mathbf{e}, 600 \mathrm{~nm}$ for $\mathbf{g}$-h. $* * * *, \mathrm{p}<0.001$. Representative images from three independent experiments are shown.

\section{Figure 6. Loss of chloride ion channels alter secretory cargo restructuring}

Confocal images of $w t$ (a) and RNAi-mediated knockdown of the intracellular channel Clic using two independent RNAi lines, $C l i c^{V D R C 105975}(\mathbf{b})$ and $C^{2}{ }^{V D R C 28303}(\mathbf{c})$ are shown. TEMs of $w t(\mathbf{f})$ and Clic ${ }^{V D R C 105975}$ (g) granules showed loss of Sgs3 filaments and loss of the complex of Sgs1 discs with the electron-dense matrix. Confocal images of RNAi-mediated knockdown of the second most abundant chloride channel gene $C l C-c$, using two independent RNAi lines, $C l C-c^{V D R C 6466}$ (d) or $C l C-c^{V D R C 106844}$ (e) results in misshapen, oblong secretory granules. TEMs of $C l C-c^{V D R C 6466}$ granules show complete disruption of the Sgs 1 discs and fragmentation of the electron-dense matrix (h). Scale bars, $10 \mu \mathrm{m}$ for a-e, $600 \mathrm{~nm}$ for $\mathbf{f}-\mathbf{h}$. Representative images from three independent experiments are shown.

\section{Figure 7. Loss of the calcium channel fwe disrupts intragranular restructuring}

The calcium channel fwe is present on secretory granule membranes as visualized in the fwe-YFP line (a). Confocal images of wt (b) and RNAi-mediated knockdown of fwe using two independent RNAi lines, 
$f w e^{V D R C 39596}$ (c) or $f w e^{G L 01498}$ (d) resulted in irregular granule morphologies. TEMs show that the loss of $f w e$ (f) results in granules that are filled with disordered winding filaments relative to $w t$ (e). Scale bars, 10 $\mu \mathrm{m}$ for a-d, $600 \mathrm{~nm}$ for e-f. Representative images from three independent experiments are shown.

\section{Figure 8. Loss of mucin-type O-glycosylation alters specific intragranular structures}

TEMs of $w t$ (a) and pgant 9 mutant $\left(\operatorname{pgant}^{4} / D f(2 R)\right)$ stage 2 SGs (b-d) show highly dysmorphic granules upon loss of pgant9. The positioning of the Sgs1 discs along the electron-dense matrix is altered in pgant 9 mutants (f) relative to $w t$ (e). Morphometric analysis of Sgs3 filament spacing shows that the inter-filament distance is reduced in pgant 9 mutants when compared to $w t$ (g). Scale bars, $600 \mathrm{~nm}$ for a-d, 200nm for e,f. Representative images from three independent experiments are shown.

Figure 9. Secretory granule maturation and intragranular cargo restructuring/segregation.

Secretory granule maturation involves the growth of granules by homotypic fusion along with the regulated restructuring of proteins, which is dependent on a lowered $\mathrm{pH}$, specific changes in calium and chloride ion concentration and O-glycosylation. Restructuring of the mucins Sgs1 and Sgs3 during the maturation phase results in intragranular segregation of these distinct cargo proteins. 


\section{REFERENCES}

1. Arvan P, Castle D. Sorting and storage during secretory granule biogenesis: looking backward and looking forward. Biochem J 332 ( Pt 3), 593-610 (1998).

2. Malhotra V. Unconventional protein secretion: an evolving mechanism. EMBO J 32, 1660-1664 (2013).

3. Sossin WS, Fisher JM, Scheller RH. Sorting within the regulated secretory pathway occurs in the trans-Golgi network. J Cell Biol 110, 1-12 (1990).

4. Sossin WS, Sweet-Cordero A, Scheller RH. Dale's hypothesis revisited: different neuropeptides derived from a common prohormone are targeted to different processes. Proc Natl Acad Sci US $A$ 87, 4845-4848 (1990).

5. Fisher JM, Sossin W, Newcomb R, Scheller RH. Multiple neuropeptides derived from a common precursor are differentially packaged and transported. Cell 54, 813-822 (1988).

6. Ishida-Yamamoto A, et al. Epidermal lamellar granules transport different cargoes as distinct aggregates. J Invest Dermatol 122, 1137-1144 (2004).

7. Merighi A, Polak JM, Gibson SJ, Gulbenkian S, Valentino KL, Peirone SM. Ultrastructural studies on calcitonin gene-related peptide-, tachykinins- and somatostatin-immunoreactive neurones in rat dorsal root ganglia: evidence for the colocalization of different peptides in single secretory granules. Cell Tissue Res 254, 101-109 (1988).

8. Salio C, Averill S, Priestley JV, Merighi A. Costorage of BDNF and neuropeptides within individual dense-core vesicles in central and peripheral neurons. Dev Neurobiol 67, 326-338 (2007).

9. van Nispen tot Pannerden H, de Haas F, Geerts W, Posthuma G, van Dijk S, Heijnen HF. The platelet interior revisited: electron tomography reveals tubular alpha-granule subtypes. Blood 116, 1147-1156 (2010).

10. Merighi A. Costorage and coexistence of neuropeptides in the mammalian CNS. Prog Neurobiol 66, 161-190 (2002).

11. Merighi A. Costorage of High Molecular Weight Neurotransmitters in Large Dense Core Vesicles of Mammalian Neurons. Front Cell Neurosci 12, 272 (2018). 
12. Arias AE, Velez-Granell CS, Mayer G, Bendayan M. Colocalization of chaperone Cpn60, proinsulin and convertase PC1 within immature secretory granules of insulin-secreting cells suggests a role for Cpn60 in insulin processing. J Cell Sci 113 ( Pt 11), 2075-2083 (2000).

13. Chimienti $\mathrm{F}$, et al. In vivo expression and functional characterization of the zinc transporter ZnT8 in glucose-induced insulin secretion. J Cell Sci 119, 4199-4206 (2006).

14. Ermund A, Trillo-Muyo S, Hansson GC. Assembly, Release, and Transport of Airway Mucins in Pigs and Humans. Ann Am Thorac Soc 15, S159-S163 (2018).

15. Johansson ME, et al. Composition and functional role of the mucus layers in the intestine. Cell Mol Life Sci 68, 3635-3641 (2011).

16. Ambort D, et al. Calcium and $\mathrm{pH}$-dependent packing and release of the gel-forming MUC2 mucin. Proc Natl Acad Sci US A 109, 5645-5650 (2012).

17. Raote I, Malhotra V. Protein transport by vesicles and tunnels. J Cell Biol 218, 737-739 (2019).

18. Malhotra V, Erlmann P. The pathway of collagen secretion. Annu Rev Cell Dev Biol 31, 109-124 (2015).

19. Valentijn KM, Sadler JE, Valentijn JA, Voorberg J, Eikenboom J. Functional architecture of Weibel-Palade bodies. Blood 117, 5033-5043 (2011).

20. Arike L, Hansson GC. The Densely O-Glycosylated MUC2 Mucin Protects the Intestine and Provides Food for the Commensal Bacteria. J Mol Biol 428, $3221-3229$ (2016).

21. Hansson GC. Mucus and mucins in diseases of the intestinal and respiratory tracts. $J$ Intern Med 285, 479-490 (2019).

22. Pelaseyed T, Hansson GC. Membrane mucins of the intestine at a glance. J Cell Sci 133, (2020).

23. Nilsson HE, et al. Intestinal MUC2 mucin supramolecular topology by packing and release resting on D3 domain assembly. J Mol Biol 426, 2567-2579 (2014).

24. Javitt G, et al. Assembly Mechanism of Mucin and von Willebrand Factor Polymers. Cell 183, 717-729 e716 (2020). 
25. Trillo-Muyo S, et al. Granule-stored MUC5B mucins are packed by the non-covalent formation of N-terminal head-to-head tetramers. J Biol Chem 293, 5746-5754 (2018).

26. Ridley C, et al. Assembly of the respiratory mucin MUC5B: a new model for a gel-forming mucin. J Biol Chem 289, 16409-16420 (2014).

27. Kesimer M, Makhov AM, Griffith JD, Verdugo P, Sheehan JK. Unpacking a gel-forming mucin: a view of MUC5B organization after granular release. Am J Physiol Lung Cell Mol Physiol 298, L15-22 (2010).

28. Beckendorf SK, Kafatos FC. Differentiation in the salivary glands of Drosophila melanogaster: characterization of the glue proteins and their developmental appearance. Cell 9, 365-373 (1976).

29. Korge G. Larval saliva in Drosophila melanogaster: production, composition, and relationship to chromosome puffs. Dev Biol 58, 339-355 (1977).

30. Zhimulev IF, Kolesnikov NN. Synthesis and secretion of mucoprotein glue in the salivary gland ofDrosophila melanogaster. Wilehm Roux Arch Dev Biol 178, 15-28 (1975).

31. Mary Boyd MA. The hormonal control of salivary gland secretion in Drosophila melanogaster: Studies in vitro. Journal of Insect Physiology 23, 517-523 (1977).

32. Ashburner M, Richards G. Sequential gene activation by ecdysone in polytene chromosomes of Drosophila melanogaster. III. Consequences of ecdysone withdrawal. Dev Biol 54, 241-255 (1976).

33. Biyasheva A, Do TV, Lu Y, Vaskova M, Andres AJ. Glue secretion in the Drosophila salivary gland: a model for steroid-regulated exocytosis. Dev Biol 231, 234-251 (2001).

34. Rousso T, Schejter ED, Shilo BZ. Orchestrated content release from Drosophila glue-protein vesicles by a contractile actomyosin network. Nat Cell Biol 18, 181-190 (2016).

35. Tran DT, Masedunskas A, Weigert R, Ten Hagen KG. Arp2/3-mediated F-actin formation controls regulated exocytosis in vivo. Nat Commun 6, 10098 (2015).

36. Reynolds HM, Zhang L, Tran DT, Ten Hagen KG. Tangol coordinates the formation of endoplasmic reticulum/Golgi docking sites to mediate secretory granule formation. J Biol Chem 294, 19498-19510 (2019). 
37. Raote I, Saxena S, Campelo F, Malhotra V. TANGO1 marshals the early secretory pathway for cargo export. Biochim Biophys Acta Biomembr 1863, 183700 (2021).

38. Burgess J, et al. AP-1 and clathrin are essential for secretory granule biogenesis in Drosophila. Mol Biol Cell 22, 2094-2105 (2011).

39. Farkas R, Suakova G. Developmental regulation of granule size and numbers in larval salivary glands of drosophila by steroid hormone ecdysone. Cell Biol Int 23, 671-676 (1999).

40. Tran DT, Ten Hagen KG. Real-time insights into regulated exocytosis. J Cell Sci 130, 1355-1363 (2017).

41. Ma CJ, Brill JA. Endosomal Rab GTPases regulate secretory granule maturation in Drosophila larval salivary glands. Commun Integr Biol 14, 15-20 (2021).

42. Ma CJ, et al. An early endosome-derived retrograde trafficking pathway promotes secretory granule maturation. J Cell Biol 219, (2020).

43. Verdugo P. Supramolecular dynamics of mucus. Cold Spring Harb Perspect Med 2, (2012).

44. Korge G. Chromosome puff activity and protein synthesis in larval salivary glands of Drosophila melanogaster. Proc Natl Acad Sci U S A 72, 4550-4554 (1975).

45. Akam ME, Roberts DB, Richards GP, Ashburner M. Drosophila: the genetics of two major larval proteins. Cell 13, 215-225 (1978).

46. Velissariou V, Ashburner M. The secretory proteins of the larval salivary gland of Drosophila melanogaster: Cytogenetic correlation of a protein and a puff. Chromosoma 77, 13-27 (1980).

47. Ji S, et al. A molecular switch orchestrates enzyme specificity and secretory granule morphology. Nat Commun 9, 3508 (2018).

48. Perez-Vilar J, Olsen JC, Chua M, Boucher RC. pH-dependent intraluminal organization of mucin granules in live human mucous/goblet cells. J Biol Chem 280, 16868-16881 (2005).

49. Allan AK, Du J, Davies SA, Dow JA. Genome-wide survey of V-ATPase genes in Drosophila reveals a conserved renal phenotype for lethal alleles. Physiol Genomics 22, 128-138 (2005). 
50. Bowman EJ, Siebers A, Altendorf K. Bafilomycins: a class of inhibitors of membrane ATPases from microorganisms, animal cells, and plant cells. Proc Natl Acad Sci U S A 85, 7972-7976 (1988).

51. Steiner CA, Litt M, Nossal R. Effect of $\mathrm{Ca}++$ on the structure and rheology of canine tracheal mucin. Biorheology 21, 235-252 (1984).

52. Verdugo P, Deyrup-Olsen I, Aitken M, Villalon M, Johnson D. Molecular mechanism of mucin secretion: I. The role of intragranular charge shielding. J Dent Res 66, 506-508 (1987).

53. Ponnalagu D, et al. Molecular identity of cardiac mitochondrial chloride intracellular channel proteins. Mitochondrion 27, 6-14 (2016).

54. Guzman RE, Grieschat M, Fahlke C, Alekov AK. ClC-3 is an intracellular chloride/proton exchanger with large voltage-dependent nonlinear capacitance. ACS Chem Neurosci 4, 994-1003 (2013).

55. Li X, Wang T, Zhao Z, Weinman SA. The ClC-3 chloride channel promotes acidification of lysosomes in CHO-K1 and Huh-7 cells. Am J Physiol Cell Physiol 282, C1483-1491 (2002).

56. Yao CK, et al. A synaptic vesicle-associated $\mathrm{Ca} 2+$ channel promotes endocytosis and couples exocytosis to endocytosis. Cell 138, 947-960 (2009).

57. Syed ZA, Hard T, Uv A, van Dijk-Hard IF. A potential role for Drosophila mucins in development and physiology. PLoS One 3, e3041 (2008).

58. Wendler F, Page L, Urbe S, Tooze SA. Homotypic fusion of immature secretory granules during maturation requires syntaxin 6. Mol Biol Cell 12, 1699-1709 (2001).

59. Du W, et al. HID-1 is required for homotypic fusion of immature secretory granules during maturation. Elife 5, (2016).

60. Colomer V, Kicska GA, Rindler MJ. Secretory granule content proteins and the luminal domains of granule membrane proteins aggregate in vitro at mildly acidic $\mathrm{pH}$. $J$ Biol Chem 271, 48-55 (1996).

61. Chanat E, Huttner WB. Milieu-induced, selective aggregation of regulated secretory proteins in the trans-Golgi network. J Cell Biol 115, 1505-1519 (1991). 
62. Hutton JC. Insulin secretory granule biogenesis and the proinsulin-processing endopeptidases. Diabetologia 37 Suppl 2, S48-56 (1994).

63. Hutton JC. The insulin secretory granule. Diabetologia 32, 271-281 (1989).

64. Lidell ME, Johansson ME, Hansson GC. An autocatalytic cleavage in the $\mathrm{C}$ terminus of the human MUC2 mucin occurs at the low pH of the late secretory pathway. J Biol Chem 278, 13944-13951 (2003).

65. Lidell ME, Hansson GC. Cleavage in the GDPH sequence of the C-terminal cysteine-rich part of the human MUC5AC mucin. Biochem J 399, 121-129 (2006).

66. Singh H. Two decades with dimorphic Chloride Intracellular Channels (CLICs). FEBS Lett 584, 2112-2121 (2010).

67. Fernandez-Salas E, et al. mtCLIC/CLIC4, an organellular chloride channel protein, is increased by DNA damage and participates in the apoptotic response to p53. Mol Cell Biol 22, 3610-3620 (2002).

68. Berryman M, Bruno J, Price J, Edwards JC. CLIC-5A functions as a chloride channel in vitro and associates with the cortical actin cytoskeleton in vitro and in vivo. $J$ Biol Chem 279, 34794-34801 (2004).

69. Berry KL, Bulow HE, Hall DH, Hobert O. A C. elegans CLIC-like protein required for intracellular tube formation and maintenance. Science 302, 2134-2137 (2003).

70. Suh KS, et al. CLIC4 mediates and is required for Ca2+-induced keratinocyte differentiation. $J$ Cell Sci 120, 2631-2640 (2007).

71. Ulmasov B, Bruno J, Woost PG, Edwards JC. Tissue and subcellular distribution of CLIC1. BMC Cell Biol 8, 8 (2007).

72. Valenzuela SM, et al. Molecular cloning and expression of a chloride ion channel of cell nuclei. $J$ Biol Chem 272, 12575-12582 (1997).

73. Duncan RR, Westwood PK, Boyd A, Ashley RH. Rat brain p64H1, expression of a new member of the p64 chloride channel protein family in endoplasmic reticulum. $J$ Biol Chem 272, 2388023886 (1997). 
74. Chuang JZ, Milner TA, Zhu M, Sung CH. A $29 \mathrm{kDa}$ intracellular chloride channel p64H1 is associated with large dense-core vesicles in rat hippocampal neurons. J Neurosci 19, 2919-2928 (1999).

75. Littler DR, et al. Comparison of vertebrate and invertebrate CLIC proteins: the crystal structures of Caenorhabditis elegans EXC-4 and Drosophila melanogaster DmCLIC. Proteins 71, 364-378 (2008).

76. Hara-Chikuma M, Yang B, Sonawane ND, Sasaki S, Uchida S, Verkman AS. ClC-3 Chloride Channels Facilitate Endosomal Acidification and Chloride Accumulation. Journal of Biological Chemistry 280, 1241-1247 (2005).

77. Weinert S, et al. Lysosomal pathology and osteopetrosis upon loss of $\mathrm{H}+$-driven lysosomal Claccumulation. Science 328, 1401-1403 (2010).

78. Tran DT, Ten Hagen KG. Mucin-type O-glycosylation during development. J Biol Chem 288, 6921-6929 (2013).

79. Zhang L, Ten Hagen KG. O-Linked glycosylation in Drosophila melanogaster. Curr Opin Struct Biol 56, 139-145 (2019).

80. Bleck CKE, Kim Y, Willingham TB, Glancy B. Subcellular connectomic analyses of energy networks in striated muscle. Nat Commun 9, 5111 (2018). 


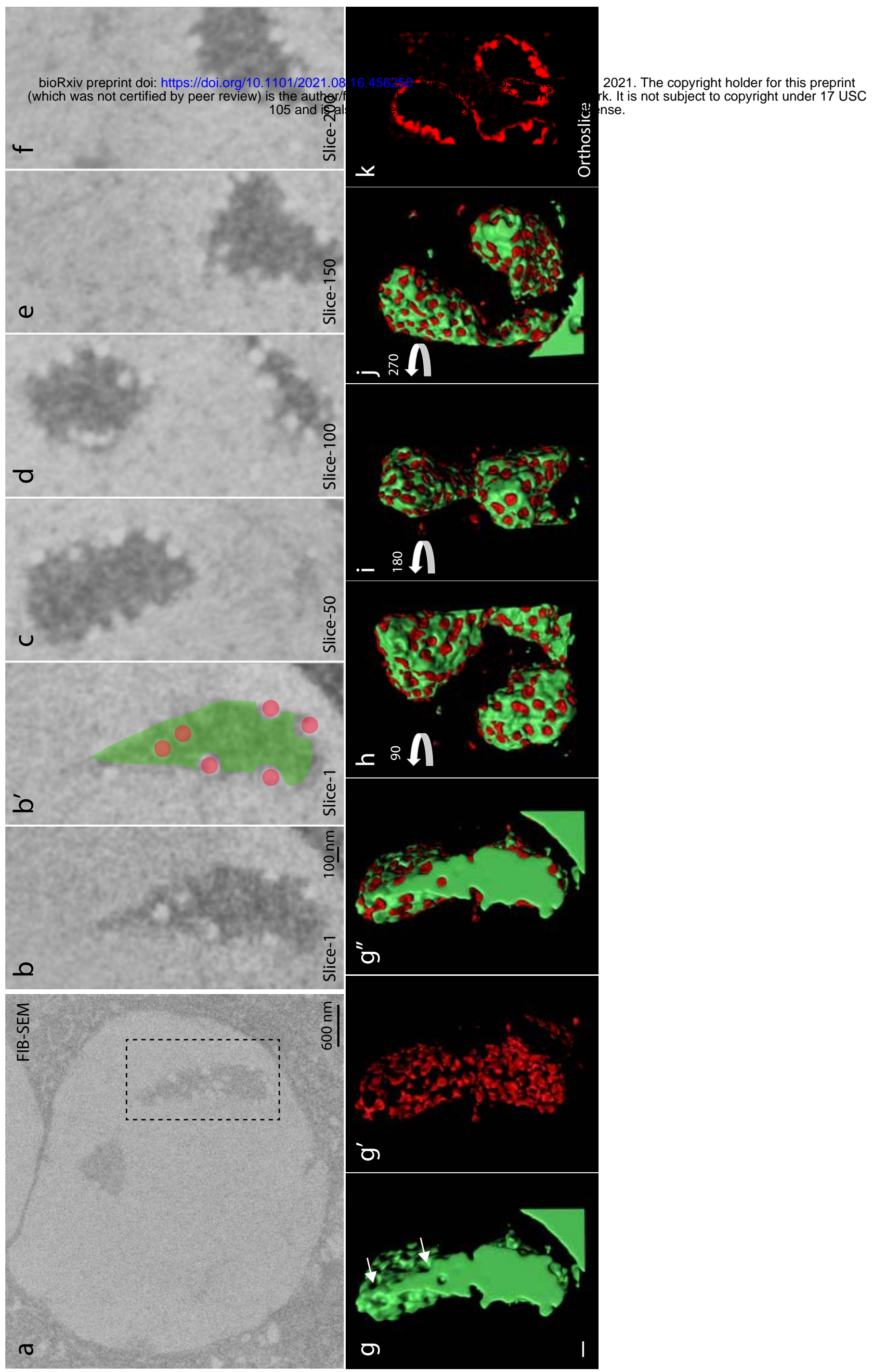




\section{Figure 3}

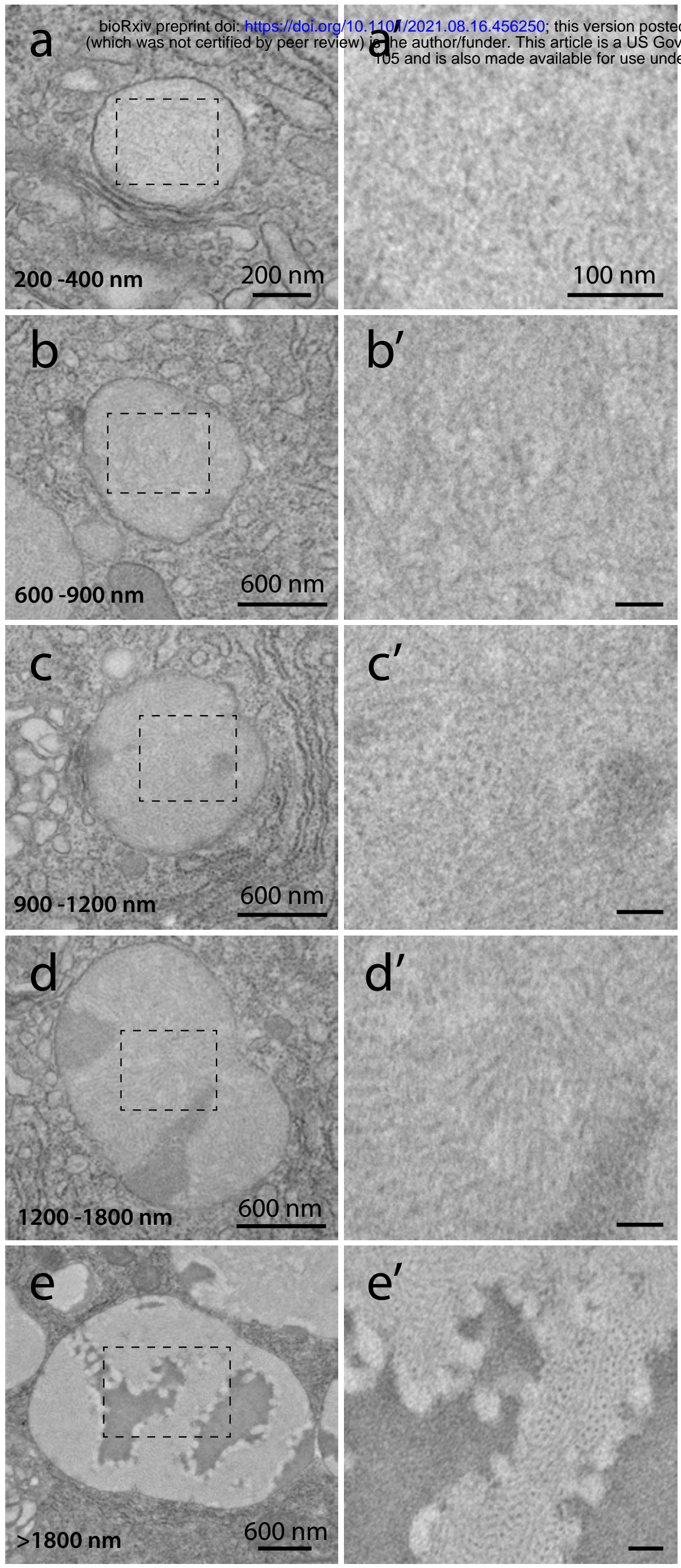




\section{Figure 4}

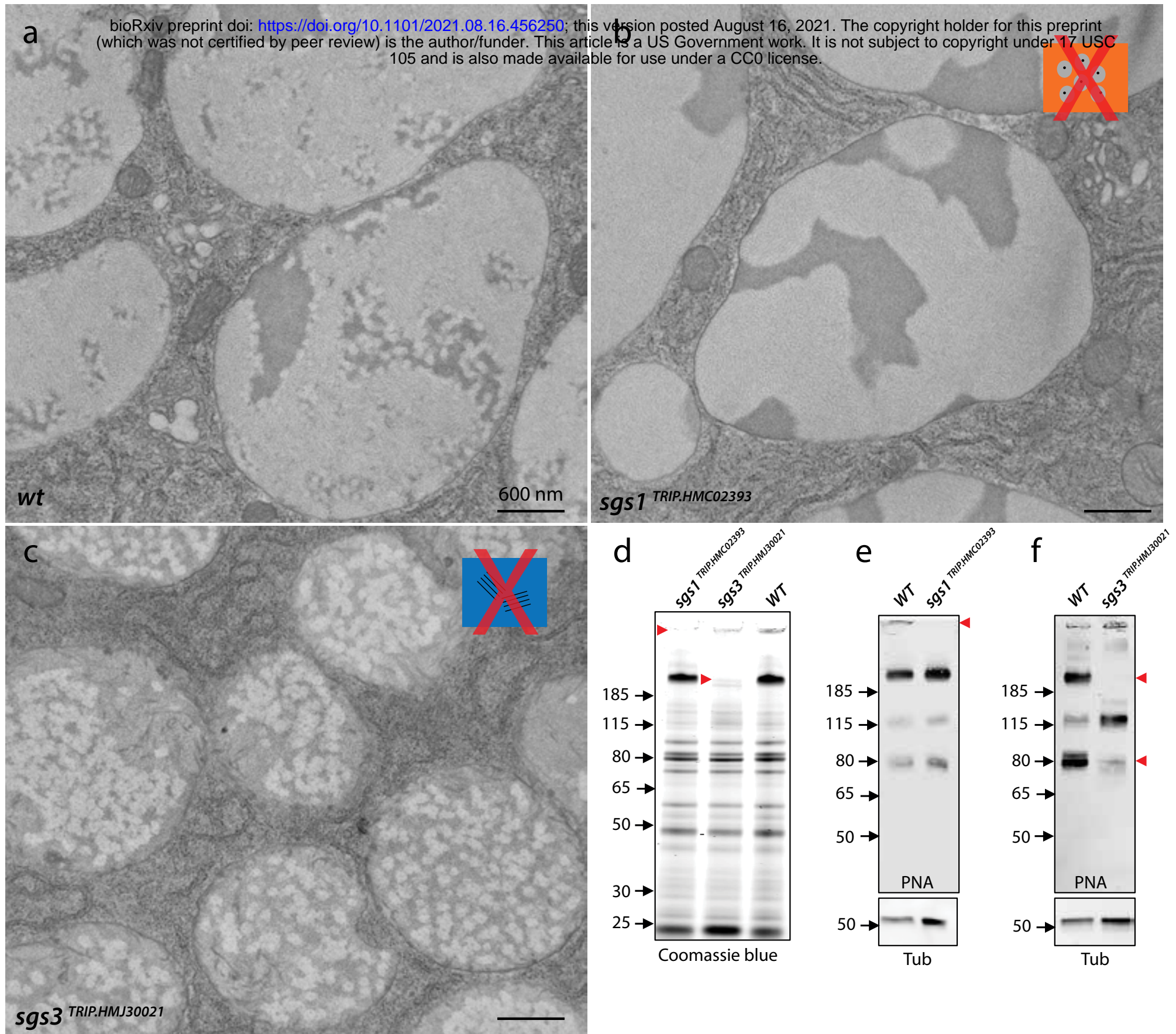




\section{Figure 5}

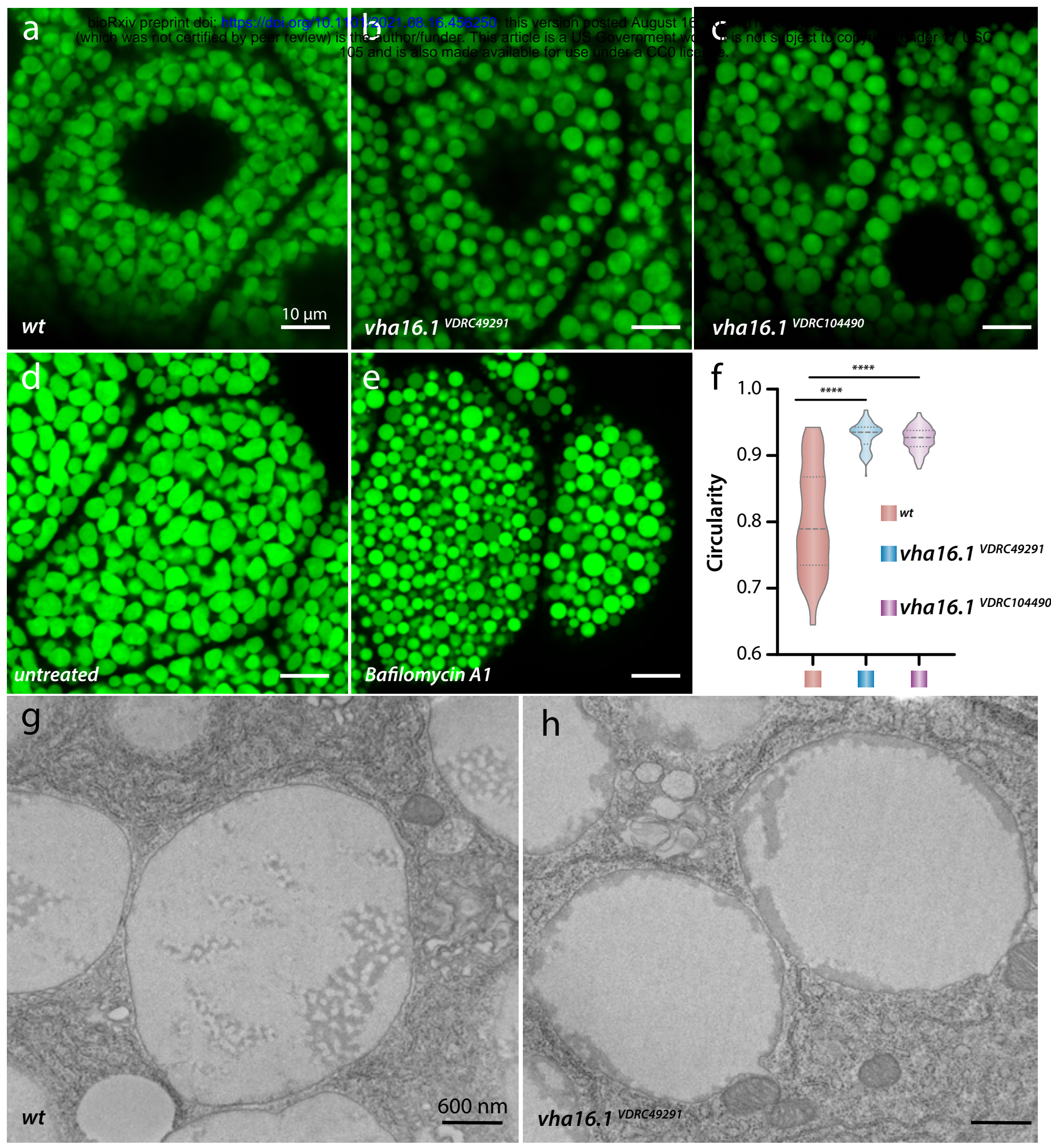



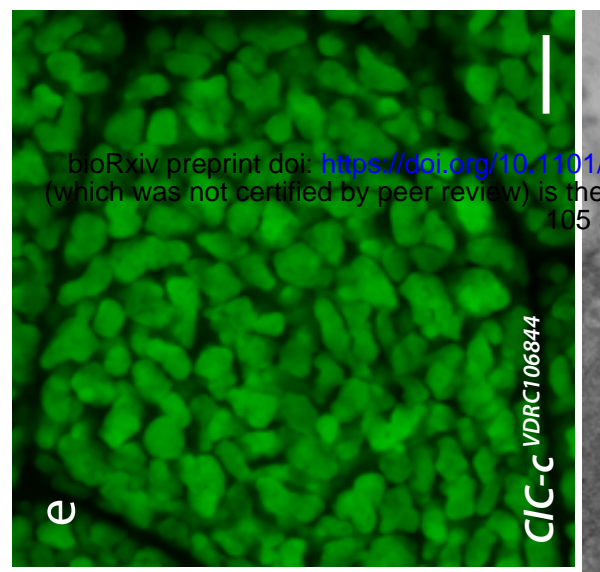

1/2021.08.16.456250; this version posted August 16, 2021. The copyright holder for this preprint 5 and is also made available for use under a CCO license.
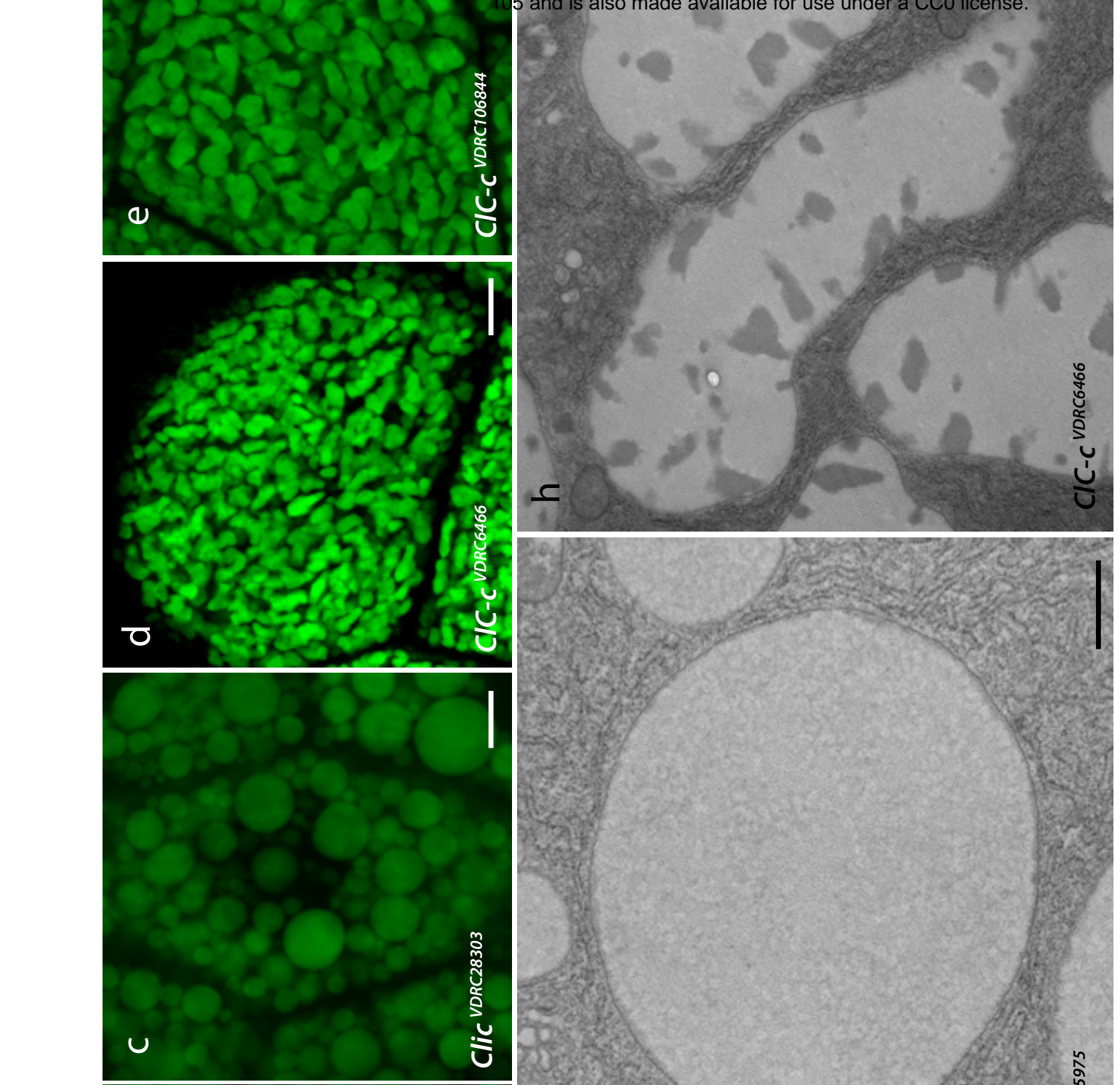

6
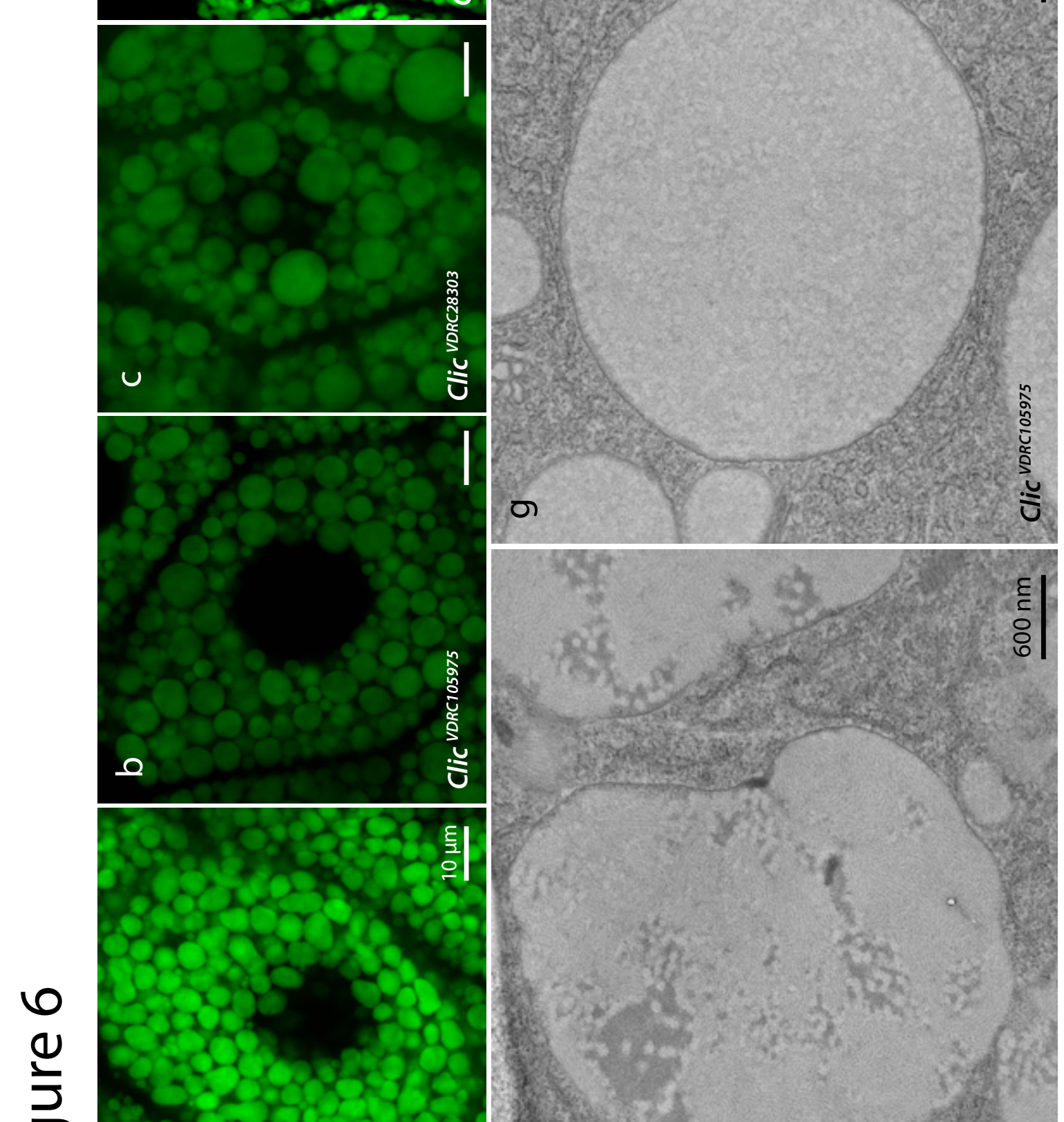
Figure 7

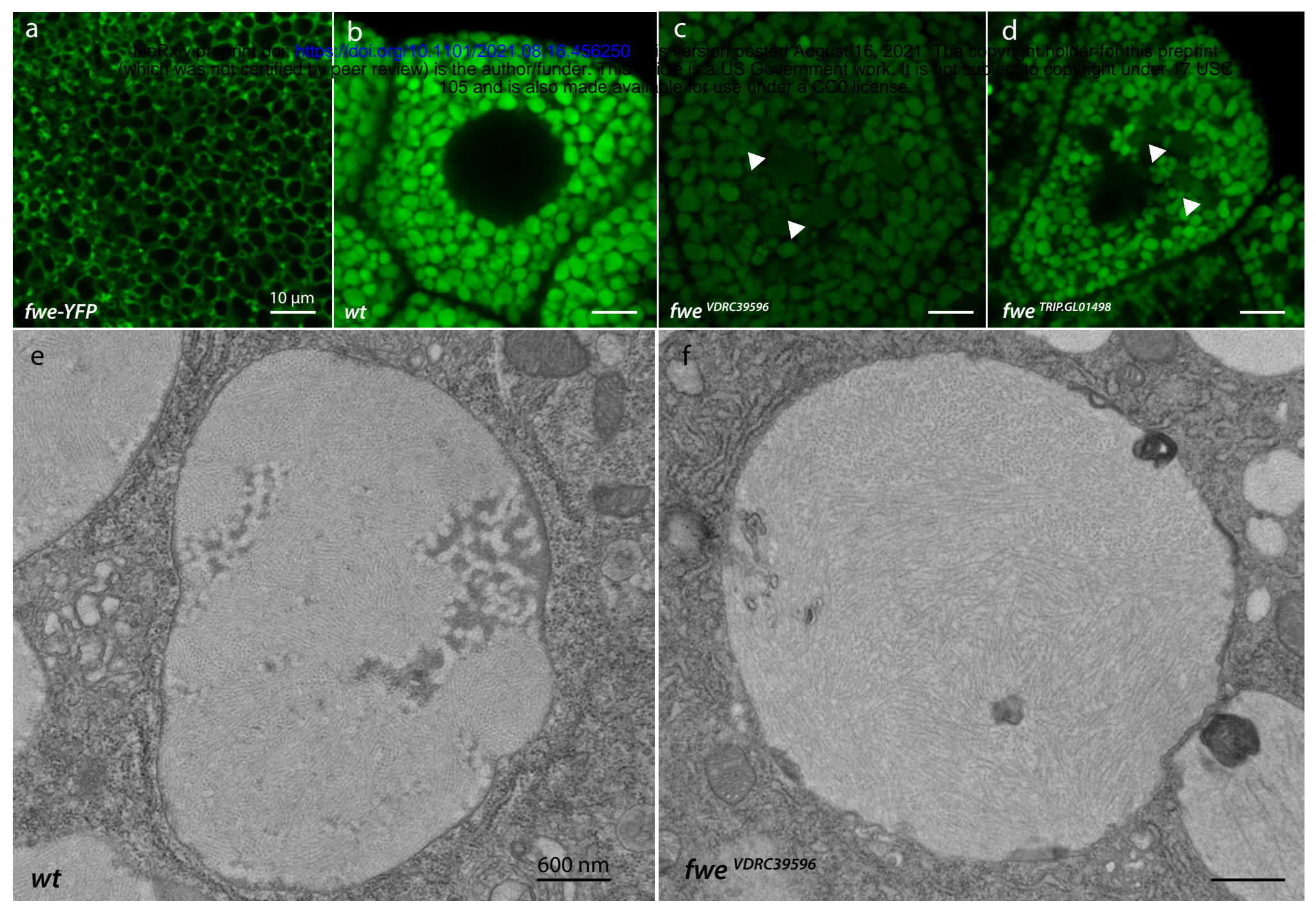




\section{Figure 8}
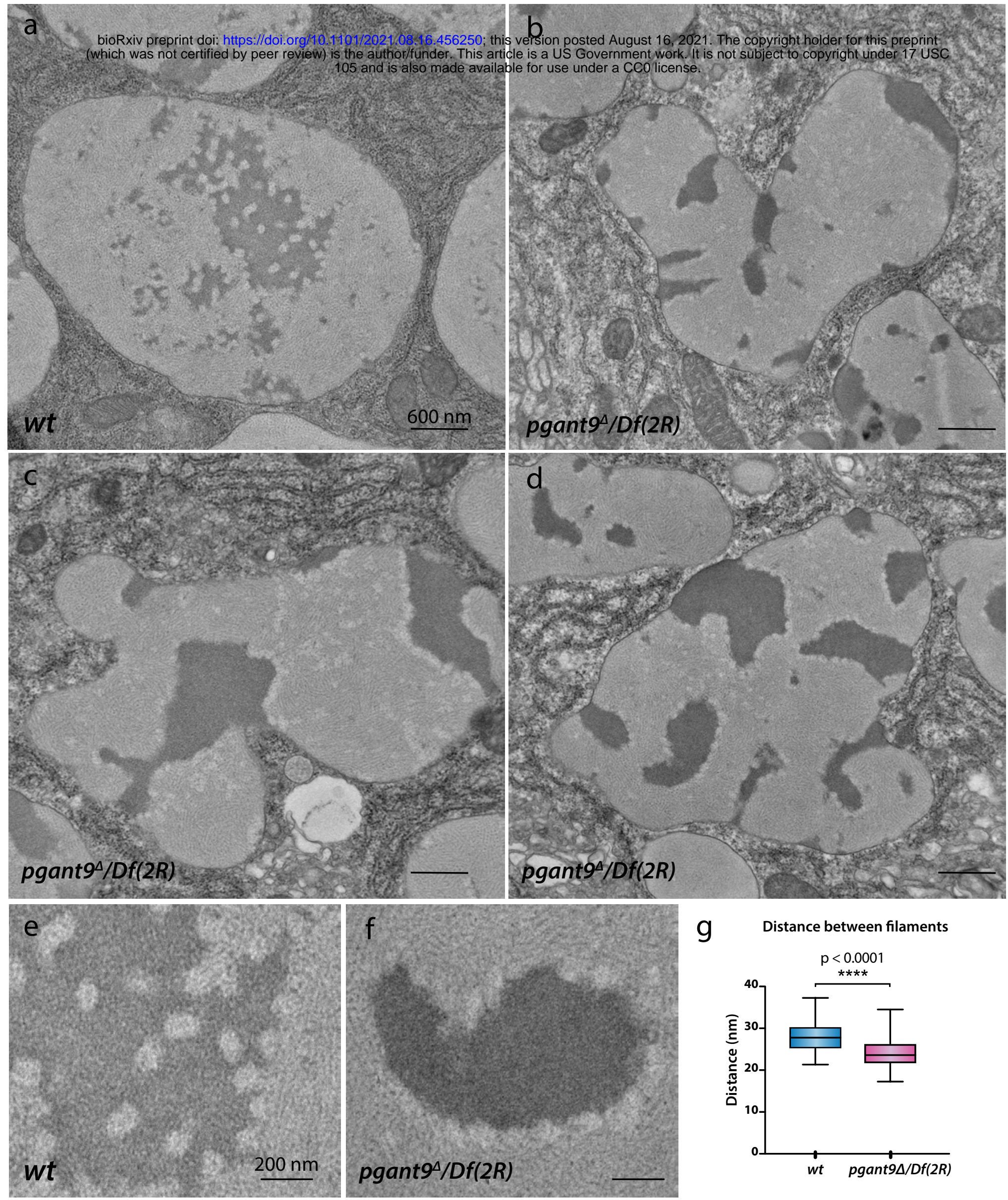

g

Distance between filaments

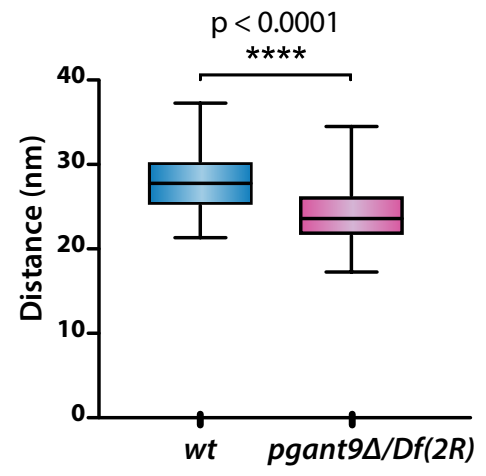




\section{Figure $9 /$ Graphical abstract}

bioRxiv preprint doi: https://doi.org/10.1101/2021.08.16.456250; this version posted August 16, 2021. The copyright holder for this preprint (which was not certified by peer review) is the author/funder. This article is a US Government work. It is not subject to copyright under 17 USC 105 and is also made available for use under a CC0 license.
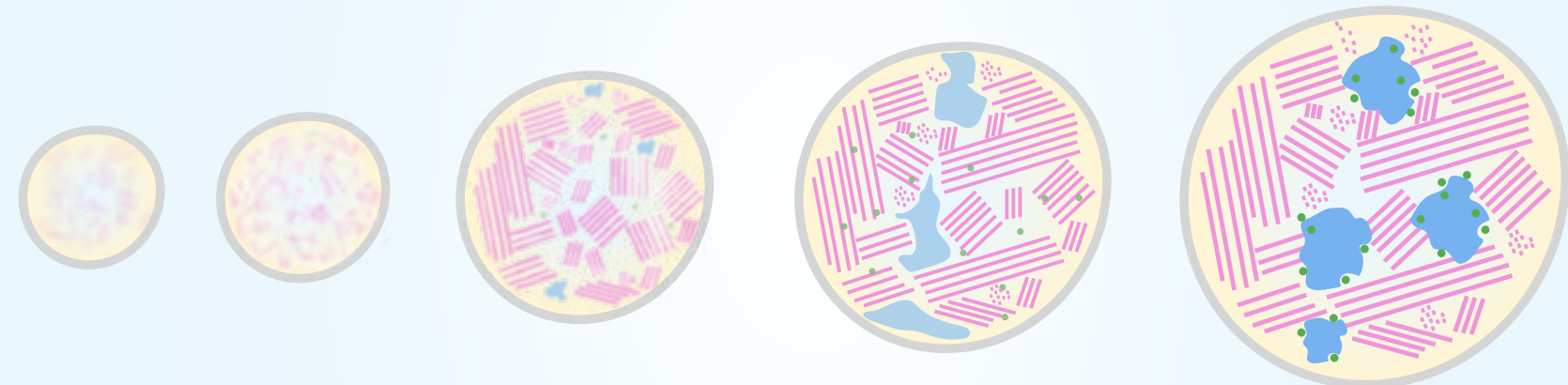

Mature granule 